\title{
Metástasis cervicales de carcinoma epidermoide de cabeza y cuello. Alternativas terapéuticas
}

\section{Cervical metastasis of squamous cell carcinoma of the head and neck. Therapeutic options}

\author{
José F. Gallegos-Hernández*, José A. Abrego-Vázquez, Aldo Olvera-Casas, Gerardo G. Minauro-Muñoz
}

y Alma L. Ortiz-Maldonado

Departamento de Tumores de Cabeza y Cuello, Hospital de Oncología, Centro Médico Nacional Siglo XXI, Instituto Mexicano del Seguro Social, Ciudad de México, México

\section{Resumen}

Introducción: Los pacientes con metástasis voluminosas de carcinoma epidermoide de cabeza y cuello representan un reto para la decisión terapéutica; se debe lograr un equilibrio entre el control oncológico y las complicaciones. La secuencia terapéutica no tiene impacto en la supervivencia, pero sí en las secuelas. Objetivo: Conocer los resultados oncológicos de pacientes con metástasis en el cuello tratados con cirugía inicial y con quimioterapia de inducción. Método: Análisis retrospectivo de pacientes sometidos a tratamiento por metástasis ganglionares cervicales de carcinoma epidermoide. La secuencia terapéutica se decidió basándose en el volumen, la resecabilidad, el sitio y el tamaño del tumor primario, y el estado general del paciente. Se calculó la supervivencia global y según el estado de portador o no del virus del papiloma humano (VPH). Resultados: Se incluyeron 30 pacientes, 22 hombres y 8 mujeres, con una media de edad de 57 años. En cuanto al estadio, 10 eran N3 y 20 eran N2. En 13 el VPH fue positivo, en 5 fue negativo y en 12 no se efectuó la determinación. Siete pacientes fueron tratados con quimioterapia inicial y 23 fueron sometidos a disección de cuello. La supervivencia a 5 años fue del $25 \%$ y el pronóstico fue mejor en los VPH positivos. Conclusión: El tratamiento de las metástasis cervicales depende de su resecabilidad. Hay que favorecer la cirugía inicial. El VPH es un factor de buen pronóstico.

PALABRAS CLAVE: Carcinoma epidermoide. Cabeza. Cuello. Disección inicial. Ganglios.

\begin{abstract}
Introduction: Patients with bulky metastases of squamous cell carcinoma of the head and neck represent a challenge for the therapeutic decision; balance between oncological control and complications should be achieved. The therapeutic sequence has no impact on survival but sequelae. Objective: We analyze the oncological results in patients with bulky metastases on the neck treated with induction chemotherapy and with up-front neck dissection. Method: Retrospective analysis of patients undergoing treatment for cervical lymph node metastasis of squamous cell carcinoma; The therapeutic sequence was decided based on volume, resectability, site, and size of the primary and general condition of the patient. Overall survival was calculated based on human papilloma virus (HPV) status. Results: There were 30 patients, 22 men and eight women with a mean age of 57 years, $10 \mathrm{~N} 3$ and $20 \mathrm{~N} 2$. In 13 the HPV was positive, in 5 negative and in 12 the determination was not made. Seven patients were treated with initial chemotherapy, and 23 underwent neck dissection; the 5-year survival was 25\%, and the prognosis was better in the positive HPV. Conclusion: The treatment of cervical metastasis depends on the possibility of resection. We should favor the initial surgery. The HPV is a factor of good prognosis.
\end{abstract}

KEY WORDS: Squamous cell carcinoma. Head. Neck. Up-front neck dissection. Nodes.

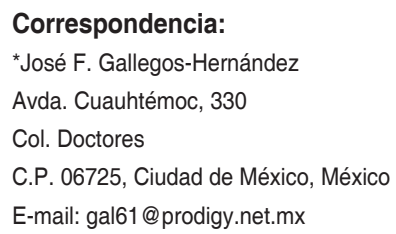

Fecha de recepción: 04-05-2018

Fecha de aceptación: 13-06-2018

DOI: 10.24875/CIRU.18000412
Cir Cir. 2019;87:141-145

Contents available at PubMed www.cirugiaycirujanos.com 


\section{Introducción}

En México, alrededor del $60 \%$ de los pacientes con cáncer de cabeza y cuello son diagnosticados en etapas avanzadas ${ }^{1}$, ya sea con tumores primarios voluminosos o con metástasis ganglionares de gran tamaño que dificultan la decisión de la secuencia terapéutica.

En ocasiones las metástasis son voluminosas, pero el tumor primario no es localizado, fenómeno conocido como metástasis cervicales con tumor primario desconocido2; en otras ocasiones, las metástasis están ocultas (no palpables) y el tumor primario es el voluminoso (T3-4), lo que sucede principalmente en tumores con poca linfofilia o facilidad para enviar metástasis al cuello.

Las metástasis ganglionares múltiples o voluminosas con tumores primarios no encontrados, pequeños o candidatos a radioterapia inicial como tratamiento representan un verdadero reto terapéutico, ya que si bien el tratamiento estándar es la radiación o la asociación de quimioterapia y radiación, la tasa de persistencia tumoral o de recurrencia es alta y hace del procedimiento quirúrgico de rescate un gesto con una alta tasa de complicaciones ${ }^{3}$, algunas de ellas mortales, como la rotura de los grandes vasos del cuello.

El tratamiento estándar de las metástasis cervicales es la disección de cuello, cuya radicalidad depende del volumen de las metástasis y de la infiltración de las estructuras no ganglionares (esternocleidomastoideo, vena yugular interna y nervio accesorio espinal). Este procedimiento permite clasificar posoperatoriamente ${ }^{4,5}$ a los pacientes y según ello decidir el tratamiento adyuvante a seguir. Sin embargo, cuando las metástasis son irresecables, o bien cuando son originadas por tumores primarios que usualmente no son tratados con cirugía (orofaringe y nasofaringe, por ejemplo), el tratamiento concomitante de quimio-radiación es el generalmente utilizado. Sin embargo, algunos pacientes sometidos a este tratamiento presentan persistencia de las metástasis cervicales y es necesario efectuar una disección radical de cuello, lo que implica una alta tasa de complicaciones posoperatorias.

Con el objeto de disminuir la tasa de complicaciones posoperatorias en la cirugía de rescate, las alternativas terapéuticas son efectuar la disección de cuello inicial y tratar el tumor primario con radioterapia o quimio-radioterapia, o bien, en pacientes con metástasis clínicamente irresecables, iniciar el tratamiento con quimioterapia de inducción y efectuar disección cervical si tras el tratamiento la respuesta ganglionar lo permite, esto es, "convertir» las metástasis no resecables en resecables y así poder ofrecer la secuencia terapéutica estándar: cirugía y radioterapia o quimio-radioterapia según el resultado histopatológico ${ }^{3}$.

Existen características de las metástasis que hacen predecir que las metástasis cervicales persistirán o tendrán una recurrencia en corto tiempo después de la radioterapia o el tratamiento concomitantes; cuando estas se presentan, se debe preferir la cirugía inicial con el objeto de una adecuada estadificación y de disminuir la tasa de complicaciones durante un eventual rescate ${ }^{6}$.

El estado del virus del papiloma humano (VPH) es un factor de buen pronóstico ya conocido ${ }^{7-9}$, sobre todo en el cáncer orofaríngeo, y aunque aún no existe evidencia suficiente para poder disminuir la intensidad del tratamiento en los pacientes VPH positivos, su presencia podría favorecer el tratamiento quirúrgico inicial o bien el tratamiento con radioterapia cuando el volumen metastásico no sea tan importante, ya que en estos pacientes la posibilidad de control es mayor.

El objetivo del presente análisis es conocer los resultados oncológicos de una serie de pacientes tratados en forma secuencial con cirugía inicial o quimioterapia de inducción seguida de cirugía en aquellos que presentaron respuesta suficiente como para ser sometidos a disección de cuello.

\section{Método}

Se efectuó un análisis retrospectivo del expediente clínico de los pacientes con diagnóstico de metástasis cervicales de carcinoma epidermoide con tumores primarios no identificados o clasificados como T1 y T2 tratados en un periodo de 7 años, de 2009 a 2015. Se evaluaron el tipo de tratamiento efectuado, la presencia o no de VPH determinada por inmunohistoquímica con P16 en los ganglios cervicales, y se calculó la supervivencia global de la serie y según el estado de VPH (positivo o negativo).

Tras el diagnóstico histológico, los pacientes fueron evaluados clínicamente y se decidió si eran candidatos a cirugía inicial (disección radical de cuello) o si los tumores eran irresecables; los criterios de irresecabilidad fueron clínicos y se basaron en la extensión tumoral cervical, la fijación de las metástasis a la fascia cervical profunda o la fascia prevertebral, la infiltración clínica o iconográfica del eje vascular yugulo-carotídeo, la infiltración bilateral del piso de la boca y de la orofaringe, y la infiltración masiva de la piel del cuello o facial. 


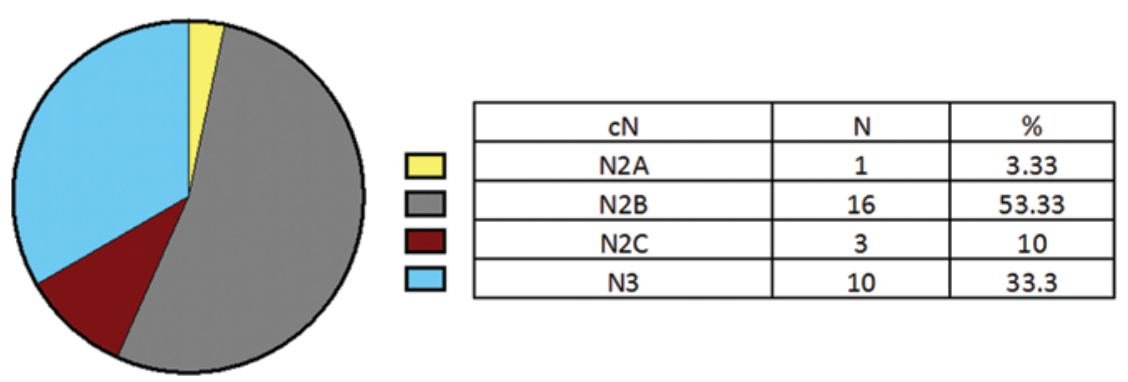

Figura 1. La mayoría de los pacientes fueron diagnosticados con metástasis cervicales múltiples menores de $6 \mathrm{~cm}$ ( $\mathrm{cN} 2)$, lo que representa el 68.6\%; el 31.4\% tuvieron metástasis mayores de $6 \mathrm{~cm}$ (cN3); $n=30$.

Los pacientes candidatos a cirugía inicial fueron clasificados de acuerdo con los resultados de patología de la disección ganglionar y recibieron tratamiento posterior con radioterapia o con quimio-radioterapia. Los pacientes considerados irresecables recibieron dos ciclos de quimioterapia sistémica basada en platino, y posterior al segundo ciclo se reevaluaron clínicamente; si la respuesta era suficiente para ser operados, se sometieron a disección radical de cuello seguida de radioterapia o tratamiento concomitante con quimio-radioterapia, y si continuaban siendo inoperables, el tratamiento a seguir era con radioterapia.

\section{Resultados}

Se incluyeron 30 pacientes, 22 hombres y 8 mujeres, con una media de edad de 57 años; 20 fueron clasificados como N2 y 10 como N3 (Fig. 1).

En 18 pacientes pudo efectuarse el análisis de p16 en muestras de los ganglios cervicales, en 12 no se pudo realizar y el estado del VPH permaneció desconocido. En el $72 \%$ de los pacientes el estado de VPH fue positivo, en el $28 \%$ fue negativo y en el $40 \%$ (12/30 pacientes) fue desconocido por no haberse efectuado la prueba.

El sitio más frecuente de localización del tumor primario fue la orofaringe, en 17 pacientes (56\%), seguido de primario desconocido en 6 (20\%). La distribución de las localizaciones puede verse en la tabla 1.

En la evaluación inicial, 23 pacientes fueron candidatos a cirugía y se sometieron a disección radical de cuello; no hubo mortalidad perioperatoria ni complicaciones mayores en este grupo de pacientes.

Siete pacientes fueron considerados irresecables por los criterios antes mencionados en el momento del diagnóstico y recibieron dos ciclos de quimioterapia de inducción basada en cisplatino.

Los siete pacientes toleraron adecuadamente el tratamiento de inducción, y seis tuvieron respuesta tal que permitió la cirugía (85\%) y fueron sometidos a disección
Tabla 1. Localización del tumor. En el $53 \%$ de los pacientes el tumor se origina en alguno de los subsitios de la orofaringe, principalmente en la base de la lengua; seis pacientes (20\%) tuvieron un tumor primario desconocido

\begin{tabular}{lll}
\hline Sitio del tumor & N & $\%$ \\
\hline Primario desconocido & 6 & 20 \\
Amígdala & 3 & 10 \\
Zona de tres repliegues & 1 & 3.3 \\
Base de la lengua & 7 & 23.3 \\
Velo palatino & 1 & 3.3 \\
Pliegue amigdalogloso & 1 & 3.3 \\
Laringe supraglótica & 1 & 3.3 \\
Epilaringe & 1 & 3.3 \\
Pared interna del seno piriforme & 3 & 10 \\
Pared lateral orofaríngea & 3 & 10 \\
Pared lateral del seno piriforme & 3 & 10 \\
\hline
\end{tabular}

radical de cuello después del segundo ciclo; un paciente presentó persistencia del tumor y siguió considerándose irresecable, por lo que fue sometido a quimio-radioterapia concomitante y posterior a este tratamiento tuvo persistencia y se le realizó cirugía de rescate.

Ninguno de los pacientes sometidos a cirugía tuvo complicaciones mayores (absceso cervical, dehiscencia de colgajos, rotura de vasos mayores del cuello, infección de herida quirúrgica, reintervención quirúrgica ni hematoma); el paciente sometido a cirugía de rescate (posterior a quimio-radioterapia concomitante) presentó infección cervical, sangrado y formación de hematoma, y fue reintervenido en dos ocasiones. No hubo mortalidad operatoria.

La supervivencia global de la serie fue del 25\% (Fig. 2). Los pacientes VPH positivos tuvieron un pronóstico mejor, con una supervivencia a 5 años del $45 \%$, en forma significativa al compararlos con los pacientes con VPH negativo o desconocido (Fig. 3). 


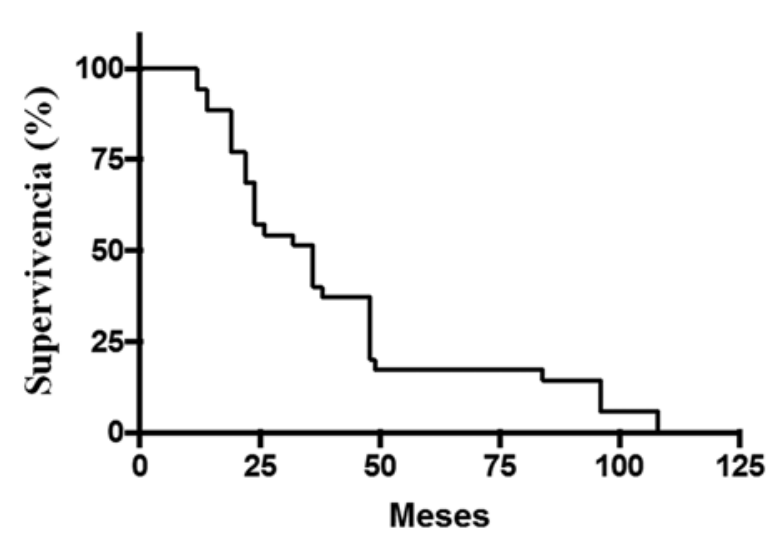

Figura 2. La supervivencia a 5 años de la totalidad de los pacientes es del $25 \%$, debido a la etapa avanzada de la enfermedad en la que se efectúa el diagnóstico.

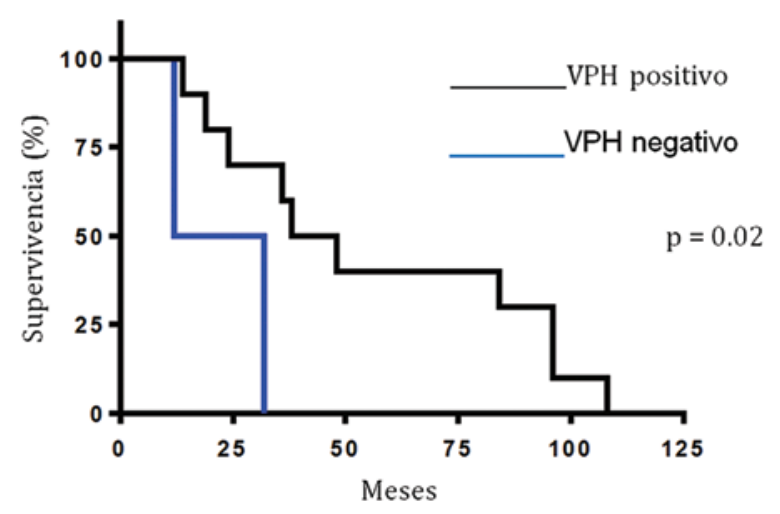

Figura 3. La presencia de virus del papiloma humano (VPH) en los ganglios metastásicos es factor de buen pronóstico en comparación con los pacientes que son VPH negativos.

\section{Discusión}

Los pacientes con metástasis múltiples o voluminosas en el cuello y con tumores primarios no encontrados o candidatos a radioterapia reciben como tratamiento estándar radioterapia radical o quimioterapia y radioterapia concomitantes; no obstante, se estima que aproximadamente el $25 \%$ de estos pacientes presentan persistencia tumoral al tratamiento o tienen una recurrencia temprana (en el primer año tras finalizar el tratamiento), y requieren cirugía de rescate. Este procedimiento se asocia a una alta tasa de complicaciones posoperatorias ${ }^{10}$, de tal manera que es preferible efectuar el procedimiento quirúrgico de forma inicial con el objeto de disminuir la tasa de complicaciones ${ }^{11}$. Sin embargo, no en todos los pacientes puede hacerse el procedimiento quirúrgico en el momento del diagnóstico; en ocasiones, las metástasis forman conglomerados que infiltran la fascia prevertebral o el eje vascular yugulo-carotídeo, o están fijas a estructuras profundas del cuello y por lo tanto son consideradas irresecables.

Los pacientes con metástasis irresecables tienen tres opciones terapéuticas: radioterapia radical, quimio-radioterapia concomitante (considerada el tratamiento estándar en pacientes con cáncer avanzado de cabeza y cuello), o quimioterapia de inducción o neoadyuvante; usualmente se administran dos ciclos incluyendo en el esquema una sal platinada, y posterior a su administración se efectúa una evaluación clínica, en la cual, si se considera que es posible realizar cirugía, esta se lleva a cabo, y de no haber respuesta suficiente debe continuarse con el tratamiento establecido, ya sea radioterapia o quimio-radioterapia ${ }^{12}$.

En la presente serie encontramos una respuesta importante al tratamiento de inducción (85\%) que permitió efectuar cirugía posterior. Si bien el control locorregional y la supervivencia no cambian con la secuencia terapéutica, la tasa de complicaciones es considerablemente mayor. En la presente serie no hubo complicaciones mayores en el grupo de pacientes sometidos a cirugía inicial ni en los que fueron operados después de haber recibido quimioterapia neoadyuvante. El único paciente que fue sometido a cirugía de rescate posterior a quimio-radioterapia presentó complicaciones importantes que requirieron reintervención quirúrgica.

Con estos resultados observamos que la tasa de respuesta a la quimioterapia de inducción es alta, que un porcentaje importante de pacientes inicialmente no resecables pueden ser operados tras la inducción, y que si bien no podemos demostrar que la supervivencia sea diferente, la tasa de complicaciones es mayor en los pacientes que son sometidos a cirugía de rescate cuando se comparan con los que son operados inicialmente, de tal manera que la cirugía inicial en los pacientes con metástasis resecables y la disección cervical antes de la radioterapia en los pacientes que tienen una respuesta suficiente a la quimioterapia de inducción son alternativas terapéuticas útiles cuando hay metástasis voluminosas cervicales de carcinoma epidermoide originado en las mucosas de la cabeza y el cuello.

El estado del VPH es importante, ya que no solo impacta en el pronóstico de los pacientes sino que podría, en un futuro mediato, seleccionar el tipo de tratamiento. Debido al buen pronóstico que tienen los pacientes VPH positivos no consumidores de alcohol ni tabaco, su tratamiento podría ser menos radical y ser candidatos solo a cirugía o solo a radioterapia 
dirigida al cuello y a la hemiorofaringe, con lo que la tasa de complicaciones y secuelas sería menor ${ }^{13}$.

En la presente serie, la supervivencia de los pacientes VPH positivos fue significativamente mejor en comparación con los negativos y con aquellos de los que se desconocía el estado de VPH. La supervivencia global es pobre debido a la etapa avanzada de la enfermedad en el momento del diagnóstico.

\section{Conclusiones}

El abordaje terapéutico de los pacientes con cáncer de cabeza y cuello metastásico a ganglios depende de la etapa, la resecabilidad ganglionar, el estado del paciente y el sitio anatómico de origen del tumor. Se debe de lograr un equilibrio entre el control oncológico y las complicaciones. La secuencia terapéutica no influye en la supervivencia, pero sí en las secuelas que el tratamiento deja ${ }^{14-16}$.

En los pacientes con metástasis cervicales de carcinoma epidermoide, la cirugía inicial es preferible aunque el tumor primario sea tratado de otra forma (radioterapia o quimio-radioterapia), con el objeto de disminuir la posibilidad de que se requiera cirugía posterior (de rescate) e incrementar las complicaciones. En los pacientes con metástasis irresecables, el tratamiento estándar es la quimio-radioterapia concomitante, pero en aquellos en los que clínicamente pudiese ser posible la cirugía una alternativa es la quimioterapia neoadyuvante con revaloración posterior, y efectuar la cirugía al terminar la inducción, si es factible.

Los pacientes con metástasis asociadas a infección por el VPH podrían ser candidatos solo a una variedad terapéutica o bien a radioterapia que no incluya todas las mucosas del área de la cabeza y el cuello, debido al buen pronóstico que tienen comparados con los pacientes VPH negativos ${ }^{17}$.

\section{Bibliografía}

1. Gallegos-Hernández JF. El cáncer de cabeza y cuello. Factores de riesgo y prevención. Cir Cir. 2006;74:287-93.

2. Gallegos-Hernández JF. Cervical lymph node metastases of squamous cell carcinoma from unknown primary. Current diagnostic and therapeutic approach. An Orl Mex. 2016:61:57-65.

3. Elicin O, Albrecht T, Haynes AG, Bojaxhiu B, Nisa L, Caversaccio M, et al. Outcomes in advanced head and neck cancer treated with up-front neck dissection prior to (chemo)radiotherapy. Otolaryngol Head Neck Surg. 2016;154:300-8

4. Sanabria A, Silver CE, Olsen KD, Medina JE, Hamoir M, Paleri V, et al. Is selective neck dissection indicated during salvage surgery for head and neck squamous cell carcinoma? Eur Arch Otolaryngol. 2014;271: 3111-9.

5. van der Putten L, de Bree R, Kuik DJ, Rietveld DH, Buter J, et al. Salvage laryngectomy: oncological and functional outcome. Oral Oncol. 2011;47:296-301

6. Strojan P, Ferlito A, Langendijk JA, Corry J, Woolgar JA, Rinaldo A, et al. Cotemporary management of lymph node metastases from an unknown primary to the neck: a review of therapeutic options. Head Neck. 2013; 35:286-93.

7. Jouhi L, Halme E, Iriala H, Saarilahti K, Kouvunene P, Pukkila M, et al. Epidemiological and treatment-related factors contribute to improved outcome of oropharyngeal squamous cell carcinoma in Finland. Acta Oncol. 2018;57:541-51.

8. Flores-de la Torre C, Hernández-Hernández DM, Gallegos-Hernández JF Human papilloma virus in patients with epidermoid head and neck carcinoma: a prognostic factor? Cir Cir. 2010;78:221-8.

9. Husain N, Neyaz A. Human papillomavirus associated head and neck squamous cell carcinoma: controversies and new concepts. J Oral Craniofac Res. 2017;7:198-205.

10. Wopken K, Bijl HP, Langendijk JA. Prognostic factors for tube feeding dependence after curative(chemo-)radiation in head and neck cancer: a systematic review of the literature. Radiother Oncol. 2017;21:32545-8.

11. Elicin O, Nisa L, Dal Pra A, Bojaxhiu B, Caversaccio M, Schmücking M, et al. Up-front neck dissection followed by definitive (chemo)-radiotherapy in head and neck squamous cell carcinoma: rationale, complications, toxicity rates and oncologic outcomes. A systematic review. Radiother Oncol. 2016:119:185-93.

12. Patil VM, Prabhash K, Noronha V, Joshi A, Dhumal S, Arya S, et al. Neoadjuvant chemotherapy followed by surgery in very locally advanced technically unresectable oral cavity cancers. Oral Oncol. 2014;50:1000-4.

13. Liu H, Li J, Zhou Y, Hu Q, Zeng Y, Mohammadreza MM. Human papillomavirus as a favorable prognostic factor in a subset of head and neck squamous cell carcinomas: a meta-analysis. J Med Virol. 2017;89:710-25.

14. Nevens D, Duprez F, Bonte K, Deron P, Huvenne W, Laenen A, et al. Upfront vs no upfront neck dissection in primary head and neck cancer radio(chemo)therapy: tumor control and late toxicity. Radiother Oncol. 2017; $124: 220-4$

15. Tangthongkum M, Kirtsreesakul V, Supanimitjaroenporn P, Leelasawwatsuk $P$. Treatment outcome of advance staged oral cavity cancer: concurrent chemo-radiotherapy compared with primary surgery. Eur Arch Otorhinolaryngol. 2017;274:2567-72.

16. Huguet $F$, Schick $U$, Pointreau $Y$. Role of induction chemotherapy in head and neck cancer. Cancer Radiother. 2017;21:510-4.

17. Mehta V, Moore-Medlin T, Flores JM, Ma X, Ekshyyan O, Nathan CO. Survival outcomes based on systematic agent used concurrently with radiation in human-papillomavirus associated oropharyngeal cancer. Oncotarget. 2017;8:70907-15. 